\title{
Motion and Form Coherence Detection in Autistic Spectrum Disorder: Relationship to Motor Control and 2:4 Digit Ratio
}

\author{
Elizabeth Milne, ${ }^{1,5}$ Sarah White, ${ }^{2}$ Ruth Campbell, ${ }^{1}$ John Swettenham, ${ }^{1}$ Peter Hansen, ${ }^{3}$ \\ and Franck Ramus ${ }^{2,4}$
}

\begin{abstract}
Children with autistic spectrum disorder and controls performed tasks of coherent motion and form detection, and motor control. Additionally, the ratio of the 2nd and 4th digits of these children, which is thought to be an indicator of foetal testosterone, was measured. Children in the experimental group were impaired at tasks of motor control, and had lower 2D:4D than controls. There were no group differences in motion or form detection. However a sub-group of children with autism were selectively impaired at motion detection. There were significant relationships between motion coherence detection and motor control in both groups of children, and also between motion detection, fine motor control and 2D:4D in the group of children with autistic spectrum disorder.
\end{abstract}

KEY WORDS: Motion detection; motor control; foetal testosterone; autistic spectrum disorder.

The recent approach of investigating sensory abnormalities in autism has revealed a systematic impairment of motion perception in autism. Abnormal response to visual motion was first reported by Gepner et al., who found that children with autism were less posturally reactive to motion information carried in optic flow than controls (Gepner \& Mestre, 2002a, b; Gepner, Mestre, Masson, \& De-Schonen, 1995). This result has been followed by additional data, which suggests that children with autism are impaired at recognizing biological motion (Blake, Turner, Smoski, Pozdol, \& Stone, 2003) and in

\footnotetext{
${ }^{1}$ Department of Human Communication Science, UCL, London, UK.

${ }^{2}$ Institute of Cognitive Neuroscience, UCL, London, UK.

${ }^{3}$ Laboratory of Physiology, Oxford, UK.

${ }^{4}$ Laboratoire de Sciences Cognitives et Psycholinguistique, EHESS/CNRS/ENS, Paris, France.

${ }^{5}$ Correspondence should be addressed to: Elizabeth Milne, Department of Psychology, The University of Sheffield, S10 2TP, Sheffield, UK; e-mail: E.Milne@Sheffield.ac.uk
}

detecting coherent motion from noise (Milne et al., 2002; Spencer et al., 2000). Some researchers have postulated that abnormalities in specific areas of the visual system, namely the dorsal stream or magnocellular system, may give rise to motion detection impairments (Milne et al., 2002; Spencer et al., 2000). However this interpretation has been challenged by Bertone, Mottron, Jelenic, and Faubert (2003) who have reported that motion detection abnormality in autism occurs only in the detection of second order and not first order motion (second order motion is apparent from second order characteristics of a stimulus such as contrast and flicker whereas first order motion is defined by spatiotemporal changes in luminance). Bertone et al. suggest that this second, but not first, order motion impairment arises from a reduced ability to integrate complex perceptual information rather than a specific area of deficit.

Investigating motion perception in autism has only recently emerged as an important research area therefore the causes and implications of such an 
impairment are not yet fully understood. One important observation that should be recognized at the outset, however, is that abnormal motion detection is neither a necessary nor sufficient occurrence in autism. Our previous study of motion detection thresholds in autism (Milne et al., 2002) showed a significant group difference between children with autism and matched controls but also showed extensive variability in the autism data, as some children with autism showed high motion coherence thresholds (indicating poor motion detection), whereas others performed as well as the matched controls. Additionally, it is not the case that abnormal motion detection occurs only in the developmental disorder of autism. Deficits in motion perception have also been reported in dyslexia (Eden et al., 1996; Hansen, Stein, Orde, Winter, \& Talcott, 2001; Talcott et al., 1998) and in William's syndrome (Atkinson et al., 1997, 2003). It is now apparent that the deficit does not characterize all children with these disorders, but occurs in clusters of individuals within the disorders. For example, Atkinson et al. (2003) report a subgroup of children with Williams syndrome who show poor motion detection, and Ramus re-analysed individual data from seven recent studies investigating visual impairments in dyslexia, and found only $29 \%$ of the people with dyslexia in these studies showed elevated visual thresholds (Ramus, 2003). Nevertheless, the occurrence of such visual impairments appears to be higher in developmentally disordered groups than in controls.

In the study reported here, we address the issue of individual differences within the data by considering other neuro-biological correlates that might contribute to the variability in coherent motion detection. One such correlate is the ratio of fourth to second finger length (2D:4D), which has been suggested by Manning et al. to be an indicator of foetal testosterone (Manning, Scutt, Wilson, \& Lewis-Jones, 1998). A second correlate is performance on motor control and balance tasks, which provide a putative index of cerebellar function.

The suggestion that a low ratio between the 2nd and 4 th digits (i.e. longer 4 th or ring fingers than 2 nd) reflects high levels of foetal testosterone is based on the finding that low 2D:4D is a characteristic of men compared with women (Manning et al., 1998). It has long been known that the relative length of these digits is close to 1.0 in women but is lower in men (Phelps, 1952). This has been experimentally recorded in a number of number of recent studies (Brown, Hines, Fane, \& Breedloves, 2002; Manning, 2002;
Manning, Baron-Cohen, Wheelwright, \& Sanders, 2001; Ökten, Kalyoncu, \& Yaris, 2002), and has also been demonstrated in metacarpal length of male and female baboons (McFadden \& Bracht, 2003). Additionally, 2D:4D correlates with testosterone concentration in adult males (Manning et al., 1998), and masculinised (lower) 2D:4D is found in females with congenital adrenal hyperplasia, a genetic disorder which leads to high levels of prenatal androgens (Brown et al., 2002). A possible link between 2D:4D and motion perception has been suggested (Ramus, 2002) based on the understanding that foetal testosterone is known to mediate aspects of cortical development, and has been implicated in the development of sex differences in cognition and the development of cerebral laterality (Geschwind \& Galaburda, 1985). This in turn may be under direct genetic control, possibly in association with genes responsible for the growth of digits and for the differentiation of the genital bud (Hox genes: (Kondo, Zakany, Innis, \& Duboule, 1997). In rodent models, Rosen et al. have reported that the presence of foetal testosterone can mediate changes in connectivity, which occur after damage during brain development (Rosen, Herman, \& Galaburda, 1999). In these models, where cortical lesions (e.g. ectopias) are experimentally induced, excessive testosterone impacts on the development of magnocells in the rodent thalamus (Herman, Galaburda, Fitch, Carter, \& Rosen, 1997). There is neurophysiological evidence, which suggests that some people with dyslexia have magnocellular abnormality. For example, Livingstone et al. reported autopsy data from 5 dyslexic brains in which the magnocellular layers in the lateral geniculate nucleus were disordered, and the magnocells themselves were $20 \%$ smaller than in control brains (Livingstone, Rosen, Drislane, \& Galaburda, 1991). These data have been coupled with evidence of poor motion detection in dyslexia leading to the claim that impairment in the magnocellular visual system give rise to coherent motion insensitivity in dyslexia (Stein, Talcott, \& Walsh, 2000). Galaburda suggests that high levels of foetal testosterone, co-occuring with cortical ectopias and microgyri, could give rise to these thalamic abnormalities observed in dyslexic brains (Galaburda, Menard, \& Rosen, 1994). Although there is no direct physiological evidence of magnocellular abnormality in autism, cortical migration malformations similar to those observed in dyslexic brains, have also been identified by MRI in high functioning individuals with autism or Asperger's syndrome (Berthier, Starkstein, \& 
Leiguarda, 1990; Piven et al., 1990). In addition to this significantly lower 2D:4Ds have been found in children with autism as compared to age and sexmatched controls, (Manning et al., 2001). It is therefore possible that the relatively lower 2D:4D (Manning et al., 2001) and higher motion coherence thresholds in autism (Milne et al., 2002; Spencer et al., 2000) which may indicate magnocellular abnormality are related. This prediction is tested in this study.

The second prediction is of a relationship between coherent visual motion detection and fine motor control and balance. Although it has been suggested that impairment of motor control is associated primarily with Asperger's syndrome, there is also evidence of motor impairment in children diagnosed with both autism and AS (Ghaziuddin, Butler, Tsai, \& Ghaziuddin, 1994; Manjiviona \& Prior, 1995). Cerebellar impairment has been inferred from poor performance on balance and fine motor control tasks (Fawcett, Nicolson, \& Dean, 1996), as the cerebellum is primarily involved in voluntary movement, balance and muscle-tone control (Stein \& Glickstein, 1992). Lesions of the cerebellum can also result in impaired motion detection (Ivry \& Diener, 1991; Nawrot \& Rizzo, 1995; Thier, Haarmeier, Treue, \& Barash, 1999). Specifically, it has been shown that people with midline cerebellar lesions are impaired at detecting coherent motion from displays similar to those used in studies of motion perception in developmental disorders (Nawrot \& Rizzo, 1995). Given the role of the cerebellum in postural control, it has been suggested that autism is characterised by a visual motion integration impairment, and that this may reflect defective visuo-cerebellar circuitry (Gepner \& Mestre, 2002b). Since several neuroimaging (Courchesne, 1997; Otsuka, Harada, Mori, Hisaoka, \& Nishitani, 1999) and postmortem studies (Bauman \& Kemper, 1994) have shown cerebellar abnormalities in autism, this may represent an anatomical correlate for abnormalities in balance and motor control. A second aspect of this study is therefore to measure fine and gross motor control in children with autistic spectrum disorder, and to investigate whether a relationship exists between the performance of these tasks and tasks of visual motion coherence sensitivity.

In summary, the purpose of the present study is to compare the performance of a group of children with autistic spectrum disorder with a typically developing control group on tasks of form and motion detection, tasks of fine and gross motor control, and to compare the 2D:4D of each group. In addition, the hypotheses that motor control and/or 2D:4D might be related to motion processing will be tested by correlation analysis.

\section{METHODS}

\section{Participants}

Forty-six children took part in the study, 23 in the autism group (22 males, 1 female), and 23 typically developing children (10 males and 13 females). The children in the autism group had been diagnosed as having either autism $(N=11)$, autistic spectrum disorder $(N=6)$ or Asperger's syndrome $(N=6)$ by clinicians using criteria specified in the DSM-IV prior to the start of the study and were attending schools or units for children with autism. The 6 children with autistic spectrum disorder had been described as having autistic-like social and emotional deficits, which were secondary to language impairment, or as having pervasive developmental disorder not otherwise specified (PDDNOS). Raven's (standard) progressive matrices were administered to assess non-verbal IQ (Raven, Court, \& Raven, 1988). Although this test does not generate standardised IQ scores, non-verbal IQ based on the participant's age was calculated by interpolating and extrapolating standard scores from the percentile scores given. Participant demographics are shown in Table I, which confirms no significant differences between the groups with respect to these variables.

\section{Procedure}

\section{Visual Tasks}

We followed the procedure outlined by Hansen et al to determine psychophysical thresholds for coherent motion and form detection (Hansen et al., 2001).

Motion Coherence: A standard random dot kinematogram (RDK) stimulus was used, consisting of two horizontally adjacent panels of moving dots. Each contained 300 white dots of high Michelson contrast $(\sim 90 \%)$ superimposed on the black background of the computer screen. The dots were 1 screen pixel in size, approximately $0.1 \times 0.1^{\circ}$, and each panel was rectangular, subtending $10 \times 14^{\circ}$ and separated horizontally by $5^{\circ}$. One panel contained a variable proportion of target dots that moved coherently $($ at $7 \% / \mathrm{sec})$ to either left or right over successive screen refreshes, whilst the remaining noise dots in 
Table I. Participant Demographics and Between Group Analysis of Raw Data

\begin{tabular}{|c|c|c|c|c|}
\hline Variable & $\begin{array}{c}\text { Mean Score (ASD) } \\
\text { Std. Dev, Range }\end{array}$ & $\begin{array}{l}\text { Mean Score (Control) } \\
\text { Std. Dev, Range }\end{array}$ & $t$-value & $p$-value \\
\hline Chronological Age & $\begin{array}{c}10 \text { years } 1 \text { month } \\
1 \text { year } 8 \text { months } \\
8: 0-12: 11\end{array}$ & $\begin{array}{c}10 \text { years } 3 \text { months } \\
1 \text { year } 1 \text { month } \\
8: 10-12: 4\end{array}$ & & N.S. \\
\hline Non-Verbal IQ & $\begin{array}{l}95 \\
14.2 \\
70-122\end{array}$ & $\begin{array}{c}102 \\
14.1 \\
84-130\end{array}$ & & N.S. \\
\hline Motion Coherence Threshold & $\begin{array}{c}17.09 \\
15.22 \\
6.24-55.14\end{array}$ & $\begin{array}{c}10.26 \\
4.13 \\
3.55-19.65\end{array}$ & & N.S. ${ }^{\dagger}$ \\
\hline Form Coherence Threshold & $\begin{array}{c}31.46 \\
7.52 \\
17.41-47.5\end{array}$ & $\begin{array}{c}27.5 \\
6.27 \\
16.56-48.01\end{array}$ & & N.S. \\
\hline Digit Ratio & $\begin{array}{c}0.951 \\
0.031 \\
0.85-1.00\end{array}$ & $\begin{array}{c}0.992 \\
0.034 \\
0.94-1.05\end{array}$ & 4.24 & $<0.01$ \\
\hline Bead Threading (secs) & $\begin{array}{l}69.52 \\
19.09 \\
38-122\end{array}$ & $\begin{array}{c}55.96 \\
7.21 \\
43.41-70\end{array}$ & 3.19 & $<0.01$ \\
\hline Finger-Thumb (secs) & $\begin{array}{l}22.29 \\
17.23 \\
5-49.73\end{array}$ & $\begin{array}{c}9.05 \\
2.9 \\
5.5-16.04\end{array}$ & $3.43^{\dagger}$ & $<0.01$ \\
\hline Heel-Toe walking (max steps) & $\begin{array}{r}12.35 \\
4.38 \\
1-15\end{array}$ & $\begin{array}{c}15 \\
0\end{array}$ & $3.06^{\dagger}$ & $<0.01$ \\
\hline One Leg Balance (max secs) & $\begin{array}{c}14.83 \\
6.51 \\
2.3-20\end{array}$ & $\begin{array}{c}19.31 \\
2.17 \\
10.28-20\end{array}$ & $2.97^{\dagger}$ & $<0.01$ \\
\hline
\end{tabular}

Two-tailed analyses.

N.S. $=$ not significant.

${ }^{\dagger}$ Non-parametric analysis, Mann-Whitney U test statistic reported.

the panel moved with the same speed but in a direction that randomly changed between refreshes (Brownian motion). The other panel contained only noise dots. To prevent tracking of individual dots, the lifetime for each dot was fixed at three animation frames $(85 \mathrm{~ms})$ after which time the dot was regenerated at a random position inside the same panel.

Form Coherence: The form coherence threshold task, which served as a control for general visual processing performance, was designed to be as similar as possible in application to the motion task. As before, two rectangular panels were presented side by side, matched in size and overall luminance to the motion task. Each panel consisted of 600 short, high contrast line elements, with each element being $0.4^{\circ}$ in length. In one panel there was a coherent form signal, defined by line elements that were oriented tangentially to imaginary concentric circles within an area of $8^{\circ}$ diameter. Signal coherence was varied by modifying the percentage of aligned elements. At $100 \%$ coherence therefore, all line elements within the $8^{\circ}$ boundary were perfectly aligned and the circle was easy to perceive. Elements outside the $8^{\circ}$ area were orientated randomly. In the other panel, all elements were randomly orientated. Both the motion and the form task contained a number of catch trials. These were trials in which the form or motion was presented at $75 \%$ coherence, which is considered to be easily perceivable.

Figure 1 shows a schematic illustration of stimuli from the motion and form tasks. In both images, the panel on the left shows the coherent form or motion.

\section{Motor Control Tasks}

Four behavioural tests were administered, two measures of fine motor control (bead threading, and finger-thumb manipulation), and two measures of balance (heel to toe walking, and balance on one leg). 


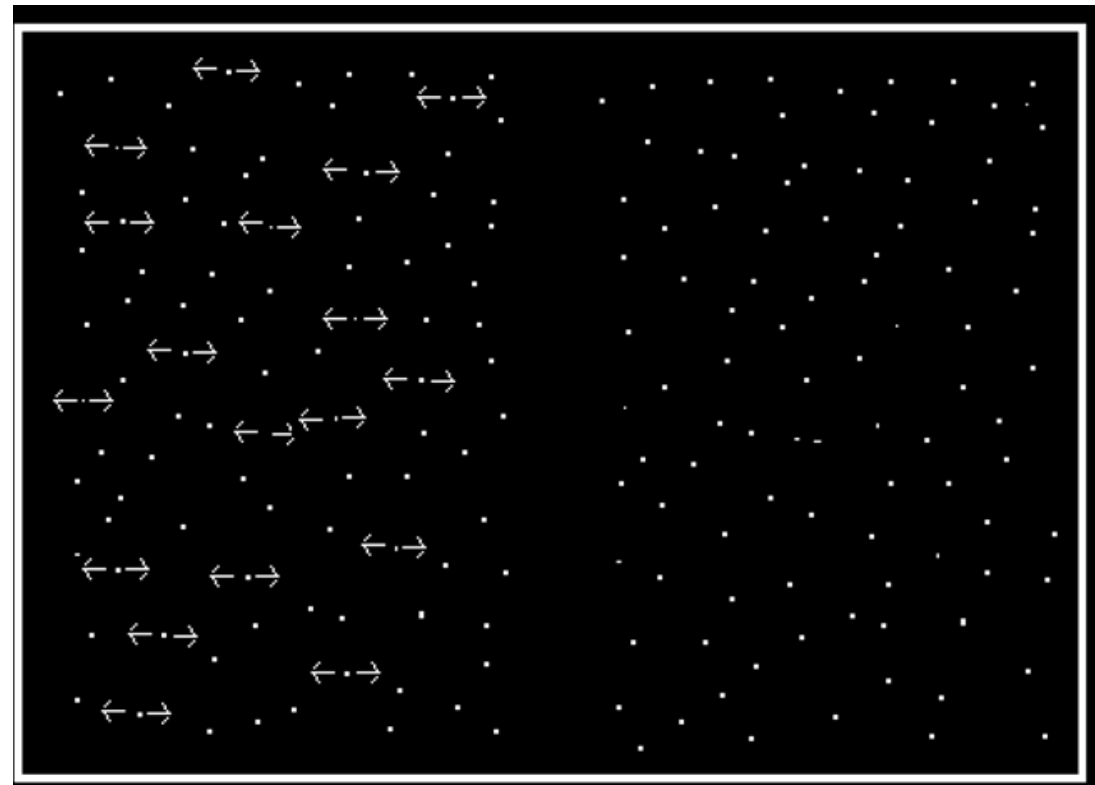

Motion detection

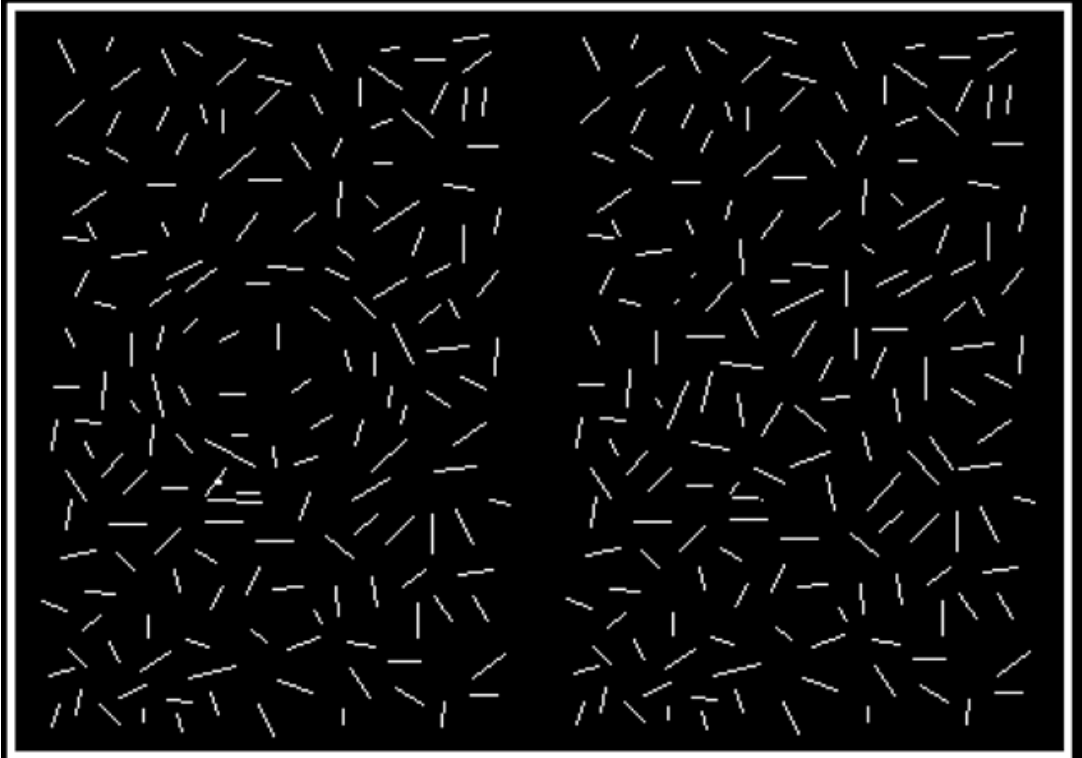

Form detection

Fig. 1. A schematic illustration of the stimuli used in the motion and form detection tasks.

Bead Threading: Fifteen large beads were strung onto a string as quickly as possible, while the child held the string in their dominant hand (Fawcett et al., 1996).
Finger and Thumb Rotation: The thumb and index finger of opposite hands were aligned thumb to finger. The lower thumb-finger pair was released and 
the hands rotated in opposite directions in order to join again at the top. The child practiced this sequence of movements until they could perform it fluently five times and then repeated it 10 times as quickly as possible whilst being timed (Dow \& Moruzzi, 1958; Fawcett et al., 1996).

Both tasks were performed twice and completion times were measured with a stopwatch. The quickest time to complete each task was recorded.

Heel-to-toe Walking: A straight, black line was positioned on the floor and the child walked along it, as if on a tightrope. They were instructed to place one foot directly in front of the other, with the toe of the back foot touching the heel of the foot in front. They walked along the line for up to a maximum of 15 steps. Any deviation from the line, or failure to connect toe to heel terminated the trial. Two attempts were given, and the maximum number of steps achieved in either trial was recorded (Henderson \& Sugden, 1992).

One Leg Balance: Each child was asked to stand on one leg, with the other foot resting on the knee of the foot that was on the ground, and with their hands on their hips, for a maximum of $20 \mathrm{sec}$. Removal of the foot from the knee or the hands from the hips, or moving the supporting foot, terminated the trial. The task was performed twice for each leg, and the average of the best times for each leg was recorded (Henderson \& Sugden, 1992).

\section{Digit Ratio}

The 2nd and 4th fingers on each child's dominant hand were measured with Vernier callipers, to $0.5 \mathrm{~mm}$. Following Manning et al. (2001), the measurement was taken from the proximal crease at the base of the finger on the palm to the fingertip of each child's hand. Digit ratio was calculated by dividing the length of the second finger by the length of the fourth finger. For one child with autism, digit length data was not obtained due to absence from school.

\section{RESULTS}

Preliminary analysis was performed to investigate if differences occurred between performance of the children diagnosed with autism $(N=11)$, autistic spectrum disorder $(N=6)$, or Asperger's syndrome $(N=6)$. Using one-way ANOVAs no significant differences were found in any of the variables (all $p>.1$ ). In the following analyses (unless otherwise stated) all the children in the experimental group were combined and are subsequently referred to as children with autism spectrum disorder (ASD).

Table I shows the mean score and standard deviation for all the variables, with $t$-values and $p$-values for comparison between the control and experimental group (parametric analysis used independent samples $t$-tests, non-parametric analyses are indicated by $\dagger$, and the Mann-Whitney U-test statistic is reported). The table shows that the groups differed significantly in digit ratio, and performance on all motor tasks.

Since 2D:4D is lower in males than in females, sex could be a confounding factor in this analysis as there were more males in the experimental group than in the control group. However, we failed to find a significant sex difference in digit ratio in the control group (10 males, mean $2 \mathrm{D}: 4 \mathrm{D}=0.986,13$ females, mean $2 \mathrm{D}: 4 \mathrm{D}=0.997, t(21)<1, p>.1)$. Additionally, when comparing the children in the experimental group (including the one female) with only the male control children, the difference between the digit ratios of the two groups remained significant, $t(30)=2.87, p<.01$.

Figure 2 presents scatterplots to illustrate the distribution of scores in the groups. For visual comparison across variables, all raw data have been converted into $z$-scores based on the mean and standard deviation of the whole sample. Single variables of fine and gross motor control were created by summing the bead-threading and finger-thumb $z$ score and the balance and heel-to-toe $z$-score respectively. Scores for the gross motor control variable, in which a high score indicates good performance, were subtracted from 0 , so in all cases a high $z$-score represents poor performance whereas a low $z$-score represents good performance. Although there was not a significant difference between the experimental and control groups in terms of motion detection, Fig. 2 shows that while the distribution of scores for the form coherence task was similar in both groups, there was a group of outliers in the sample with autism with very high (poor) motion coherence thresholds. To explore the data in terms of how many children in each group showed impairment on the motion task, deviance analysis was performed. Raw data points were converted to $z$-scores, this time based on the mean and standard deviation from the control population only. Following the procedure proposed by Ramus et al. (2003) deviant scores were 

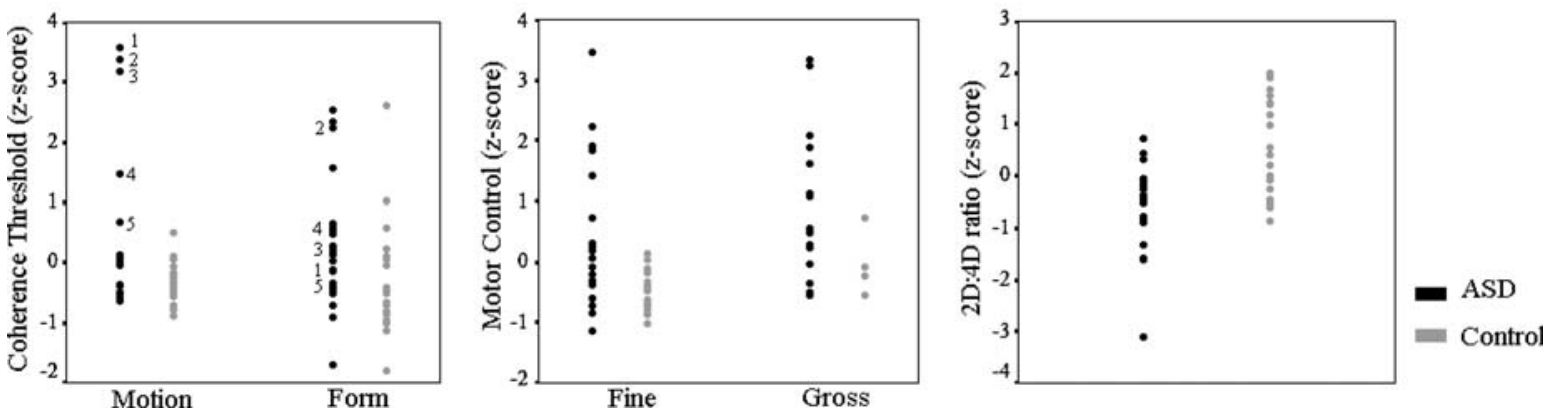

Fig. 2. Raw data points of all variables obtained by the children with ASD and typically developing children. For both the motion and form and the motor control variables, a high $z$-score indicates poor performance and a low $z$-score indicates good performance.

defined as those which were more than 1.65 standard deviations lower than the control mean. This represents the lowest $5 \%$ of the distribution generated by the typically developing children. Using this procedure, five children $(22 \%)$ in the experimental group were identified as outliers, showing particular deficit in coherent motion detection. Two of these children had diagnoses of Asperger's syndrome, one had a diagnosis of pure autism and the other two were defined as having atypical autism based on receptive language impairment and socio-emotional difficulties. The age of these participants ranged from 8 years 4 months to 11 years 10 months and the non-verbal IQ's ranged from 91 to 108. These children are labeled on the scatterplot in Fig. 2 so that their corresponding form detection scores can be identified.

\section{Investigation of Relationships Between Variables}

Bivariate correlations were performed to examine the relationships between variables. Data points for all the variables were converted to $z$-scores, based on the mean and standard deviation of the whole sample. Not all the variables were normally distributed, so Kendall's tau statistic was used to compute the correlation between variables. Twenty out of the 23 typically developing children were at ceiling on the gross motor control variable, so correlational analysis with this variable was not considered appropriate. Table II shows the two-tailed correlation matrices between the variables, which were calculated for each group separately.

To account for any effect that non-verbal IQ might exert upon the measures, we also calculated partial correlations, controlling for non-verbal IQ. Because some of the variables were abnormally distributed, we manually ranked the data in order to perform parametric partial correlations. This was done in the following way;

1. all raw data points were converted into percentiles, which creates ordinal data, and maintains the rank order of points (Lyman, 1978),

Table II. Correlation Matrix of Raw Data z-Scores (Kendal's tau, Two-tailed Analysis)

\begin{tabular}{|c|c|c|c|c|c|}
\hline & Motion & Fine Motor Control & Gross Motor Control & Digit & Form \\
\hline \multicolumn{6}{|l|}{$A S D$} \\
\hline Motion Detection & - & & & & \\
\hline Fine Motor Control & $.420 * *$ & - & & & \\
\hline Gross Motor Control & .218 & $.333^{*}$ & - & & \\
\hline Digit Ratio & $-.325^{*}$ & -.182 & -.105 & - & \\
\hline Form Detection & .281 & .277 & $.445^{* *}$ & -.039 & - \\
\hline \multicolumn{6}{|l|}{ Control } \\
\hline Motion Detection & - & & & & \\
\hline Fine Motor Control & $.325^{*}$ & & & & \\
\hline Gross Motor Control & - & - & & & \\
\hline Digit Ratio & .127 & .099 & - & - & \\
\hline Form Detection & .261 & .043 & - & .028 & - \\
\hline
\end{tabular}

$* p<.05, * * p<.01$. 
2. these percentile points were then converted into z-scores based on the mean and standard deviation of the whole population to recreate interval data, which was normally distributed and met the assumptions required for parametric analysis.

Bivariate correlations, controlling for the effect of non-verbal IQ, were performed on each group separately. This analysis generated the same results as the non-parametric analysis for the typically developing children. However the effect of partialling out nonverbal IQ revealed a significant relationship between 2D:4D and fine motor control in the children with autism, which was not apparent the non-parametric analysis when the effect of non-verbal IQ was uncontrolled. The correlation matrix of the relationship between variables after the effect of non-verbal IQ has been removed is presented in Table III. Note that a high score on both the motion and form detection tasks and on the motor tasks indicates poor performance and a low score indicates good performance.

Figure 3 shows scatter plots of the related variables. The data presented in these scatter plots has been ranked, as indicated above, in order to show the relationships between the variables regardless of the distance between points, and to standardise data across variables.

Although not the main aim of the current study, in light of existing data (Manjiviona \& Prior, 1995), an additional question of interest concerns whether the children with different clinical diagnoses differed in respect to their performance on the motor tasks. As noted above, no significant differences were seen in any of the variables between the groups of children with autism, AS or ASD. To give an indication of the number of children who obtained poorer scores on these tasks than the lowest $5 \%$ of the control distribution, deviance analysis was calculated as above for the combined variables of fine motor control and gross motor control. Using this analysis 13 out of the 23 children in the experimental group $(57 \%)$ showed impairment in fine motor control and $12(52 \%)$ showed impairment in gross motor control. This reflected impairment of fine motor control in $64 \%$ of the children with autism, $67 \%$ of the children with AS and $33 \%$ of the children with ASD, and impairment of gross motor control/balance in $45 \%$ of the children with autism, $67 \%$ of the children with AS and $50 \%$ of the children with ASD. Although this data is based on small group sizes, it never the less reflects previous data, which showed that problems of motor control occur across all groups of children on the autism spectrum.

\section{DISCUSSION}

In this study, a group of children diagnosed on the autism spectrum and a control group of typically developing children, matched for non-verbal IQ and chronological age were tested on tasks which measured coherent motion detection, coherent form detection, fine motor control and balance. The ratio between the 2 nd and 4th digits was also measured. The results of the study replicated previous data (Ghaziuddin et al., 1994; Manjiviona \& Prior, 1995; Manning et al., 2001) as the children with ASD had significantly lower digit ratios than the control group,

Table III. Partial (Two-Tailed) Correlation Matrix with the Effect of Non-verbal IQ Removed

\begin{tabular}{|c|c|c|c|c|c|}
\hline & Motion & Fine Motor Control & Gross Motor Control & Digit & Form \\
\hline \multicolumn{6}{|l|}{$A S D$} \\
\hline Motion Detection & - & & & & \\
\hline Fine Motor Control & $.587^{* *}$ & - & & & \\
\hline Gross Motor Control & .236 & $.451^{*}$ & - & & \\
\hline Digit Ratio & $-.526^{*}$ & $-.572 * *$ & -.114 & - & \\
\hline Form Detection & .368 & .308 & $.506^{*}$ & -.028 & - \\
\hline \multicolumn{6}{|l|}{ Control } \\
\hline Motion Detection & - & & & & \\
\hline Fine Motor Control & $.511^{*}$ & & & & \\
\hline Gross Motor Control & - & - & & & \\
\hline Digit Ratio & .144 & .064 & - & - & \\
\hline Form Detection & .391 & .120 & - & -.090 & - \\
\hline
\end{tabular}

Data points were ranked before analysis (see text).

$* p<.05, * * p<.01$. 

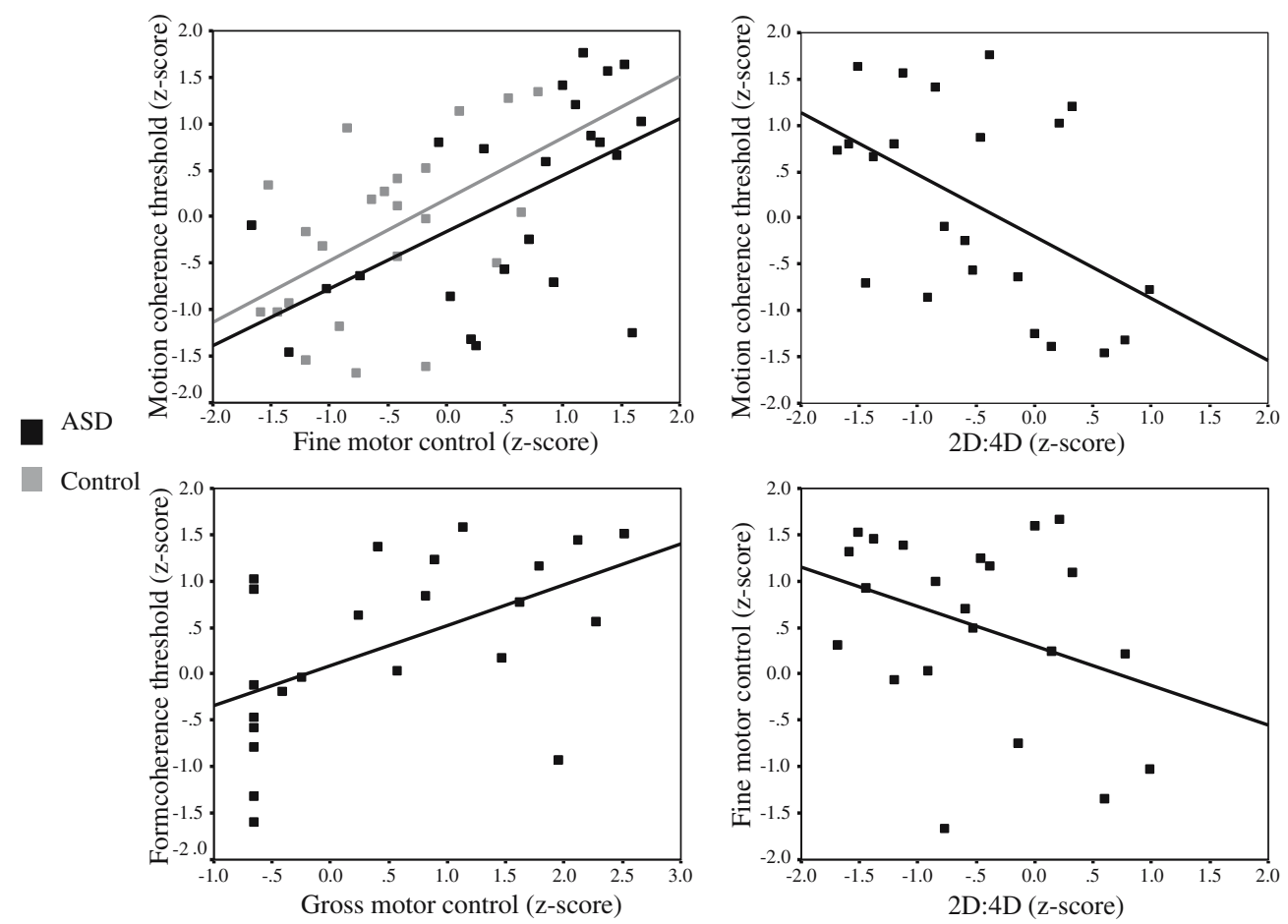

Fig. 3. Scatterplots of all related variables.

in addition to showing impairment of both fine and gross motor control. Contrary to previously published data (Milne et al., 2002; Spencer et al., 2000), there was not a significant difference in the motion coherence thresholds of the experimental and control groups, although in line with observations made in the introduction, there was a sub-group of individuals in the experimental group who had a specific impairment in detecting coherent motion with no corresponding difficulty in detecting coherent form. In addition to these group differences, significant relationships were found between visual motion processing and fine motor control in both the children with autism and the typically developing children. The children with autism also showed a relationship between visual form processing and balance, and between motion processing, fine motor control and 2D:4D. The significance of each of these findings will be discussed in turn.

The finding that the children with autism in this sample had significantly lower 2D:4D ratios than the typically developing children replicates a previous finding from Manning et al. (2001) who reported significantly lower 2D:4D in children with autism as compared to non-autistic control participants. This report is therefore the second published study to find such a difference in ASD. As discussed in the introduction, 2D:4D ratio is known to be sexually dimorphic, with males having on average higher 2D:4D ratios than females (Manning et al., 1998; Ökten et al., 2002). Although a significant sex difference of 2D:4D was not seen in the typically developing children in the sample presented here, this is thought to be due to the small sample size ( 10 males and 13 females). This finding supports the suggestion that levels of foetal testosterone are higher in autism than in controls. However, it must be noted that this suggestion remains speculative at this stage as no direct evidence of the relationship between 2D:4D ratio and foetal testosterone has been reported, and other data suggest that there is no difference between the plasma levels of testosterone in male autistic subjects as compared to controls (Tordjman et al., 1995).

This study provides further evidence that fine and gross motor control are reduced in ASD. Significant differences were found between the experimental and control groups for all four motor variables. These results mirror those of Manjiviona and Prior (1995) who tested a group of 12 children with Asperger's syndrome and 9 children with autism on the TOMI-H, a standardised test of motor 
impairment. Although it has previously been suggested that motor impairment is a clinical characteristic of AS rather than autism (Wing, 1981), Manjiviona and Prior found that $50 \%$ of the children diagnosed with AS and $66.7 \%$ of the children diagnosed with autism obtained scores which occurred in the lowest $5 \%$ of the normalised sample, indicating definite motor problems in these children. In this study we have found evidence of both fine and gross motor impairment in children diagnosed with autism, with Asperger's syndrome and with ASD. The data presented here also reflect the findings of Manjiviona and Prior in the sense that the children who were motor impaired generally showed impairment across both fine and gross motor skills. This is shown by the significant correlation between the measures of fine and gross motor skills in the experimental group. These data could reflect behavioural support for the theory of cerebellar impairment in autism.

Contrary to previous results (Milne et al., 2002; Spencer et al., 2000), motion coherence thresholds failed to distinguish the two groups statistically. In our previous study, we pointed out that not all children with autism showed elevated motion coherence thresholds, but rather that the difference in central tendency between the clinical and the control groups reflected a skewed distribution in the group with autism, with some of the children with autism (significantly more than in the control group) showing high thresholds. In the present study with a new sample of children, once again high motion coherence thresholds characterised a subset of children with autism. In this sample, the proportion of children with autism who displayed the deficit $(22 \%$ of the children were outliers as compared to the average control performance) was not enough to produce a significant mean difference between the groups. The notion of sub-groups of children showing motion detection impairment is consistent both with our previous observation of autism and also literature from other developmental disorders which outline sub-groups of impaired motion detectors with Williams syndrome and with dyslexia (Atkinson et al., 2003; Ramus, 2003). Obtaining a statistically significant difference between groups is therefore likely to be subject to sampling differences between studies, especially when working with relatively small samples of less than 30 cases per group.

Physiologically it has been shown that cells in area V5 are highly responsive to random dot kinematograms containing coherent motion and noise, and that monkeys with V5 lesion fail to extract the motion signal from noise (Britten, Shalden, Newsome, \& Movshon, 1992; Newsome \& Pare, 1988). Consequently impairment of the dorsal stream or the magnocellular system has been implicated following reports of high motion coherence thresholds (Atkinson et al., 1997; Hansen et al., 2001; Milne et al., 2002; Spencer et al., 2000; Stein \& Walsh, 1997). However, others have challenged the inference of a purely magnocellular impairment from high motion coherence thresholds (Bertone et al., 2003; Skottun, 2000), and, as was noted in the Introduction, there is no direct physiological evidence of magnocellular abnormality in autism. Recent research has highlighted the influence of attention in early vision such as stereoscopic depth (Rose, Bradshwaw, \& Hibbard, 2003) and motion perception (Chaudhuri, 1990). Therefore deficits of attention, which are known to occur in autism (Courchesne et al., 1994; Townsend, Courchesne, \& Egaas, 1996) could contribute to increased motion coherence thresholds, however the fact that the children with high motion coherence thresholds did not also have significantly higher form coherence thresholds suggests that whatever the area of deficit it is specific to the motion system. Although this paper does not advance our knowledge of the direct cause of motion impairment in autism, it provides further evidence of a sub-group of children with autism who are impaired at detecting coherent motion in the absence of a concurrent deficit in detecting coherent form, and illustrates that this impairment is related both to fine motor control and to $2 \mathrm{D}: 4 \mathrm{D}$. That is, it appears that impairments of visual motion detection are likely to co-occur with other impairments such as motor control in autism. A parallel can be drawn with data from developmental dyslexia, where abnormalities in both motion detection and motor control have been identified (Fawcett et al., 1996; Talcott et al., 1998). The existence of a relationship between motion coherence detection and 2D:4D suggests foetal testosterone may be implicated in motion detection impairments in autism. However it must be noted that the size of the groups used in correlation analysis (23) was small and therefore the confidence limits of these analyses are large.

The data also illustrate that a relationship exists between coherent motion detection and fine motor control and between coherent form detection and gross motor control. The relationship between form detection and gross motor control (balance) was not predicted. However the finding may be related to data, which illustrates a deficit in coherent form 
detection in children diagnosed with dyspraxia (O'Brien, Spencer, Atkinson, Braddick, \& WattamBell, 2002). A suggestion as to why coherent motion detection was related only to fine and not gross motor control is that good performance on these motor tasks relies on speed. Both the bead-threading and finger-thumb task measure how quickly the child can perform these tasks. The fact that poor (slow) performance on these tasks was related to poor motion detection performance suggests a common link between the system which underlies motion detection function and rapid motor generation. Since this relationship occurs in both the clinical and control group, it is likely to reflect a general developmental factor. Further research is required to determine what the common factor or factors underlying this relationship may be.

The finding that a significant relationship also exists between performance on the motor tasks and 2D:4D in the children with autism, suggests that the development of these sub-systems may be related to levels of foetal testosterone, and specifically, that anomalous development may arise from high levels of foetal testosterone. Future studies involving more children, with more sensitive measures of dorsal stream and cerebellar performance are needed to expand on the suggestions put forward here.

Genetic influences on autism do not implicate a single gene or locus of gene action, and a host of very different neurological correlates of autism have been indicated (Cody, Pelphrey, \& Piven, 2002). It seems probable that several different biological and neurological factors may contribute to the autistic syndrome; that is, it may have a heterogeneous etiology. Additionally, sensori-motor deficits have been reported in dyslexia (Fawcett et al., 1996; Livingstone et al., 1991; Lovegrove, Bowling, Badcock, \& Blackwood, 1980; Tallal, 1980), and motion detection impairments have been reported in Williams syndrome (Atkinson et al., 1997). Ramus (2002) has proposed that sensori-motor impairments are not a core feature of dyslexia, but rather secondary features arising only in cases with excessive foetal testosterone. The current data is consistent with a similar hypothesis for autism: impairment of motor control and motion detection are not inevitable in autism, but occur more frequently in the disorder than in typically developing children. If the tasks used here are to be taken as indices of magnocellular and cerebellar integrity respectively, then neither magnocellular nor cerebellar dysfunction seems to be necessary for autism to occur, but they both seem to cluster in the disorder. These will occur more frequently in children diagnosed with a developmental disorder than in typically developing children. Exploring potential sources of variation in measures of performance in people with autism, and other developmental disorders, is a useful approach to identifying contributory biological factors to these disorders, and will hopefully lead to further understanding of the neurological correlates, which underpin both the similarities and differences between developmental disorders.

\section{ACKNOWLEDGEMENTS}

We would like to thank all the children and schools who took part in this study for their time, effort and cooperation, John Manning for his discussion of 2D:4D measurement, and Marcin Szczerbinski for his discussion of statistical analysis. This research was supported by an ESRC studentship (R42200034283) and a UCL graduate school scholarship awarded to Elizabeth Milne, and partly funded by a Medical Research Council grant (G9617036) awarded to Uta Frith, and a Marie Curie fellowship of the European Community programme Quality of Life (QLGI-CT 1999-51305) awarded to Franck Ramus.

\section{REFERENCES}

Atkinson, J., Braddick, O., Anker, S., Curran, W., Andrew, R., Wattam-Bell, J., \& Braddick, F. (2003). Neurobiological models of visuospatial cognition in children with Williams syndrome: measures of dorsal-stream and frontal function. Developmental Neuropsychology, 23, 139-172.

Atkinson, J., King, J., Braddick, O., Nokes, L., Anker, S., \& Braddick, F. (1997). A specific deficit of dorsal stream function in Williams' syndrome. Neuroreport, 8, 1919-1922.

Bauman, M. L., \& Kemper, T. L. (1994). Neuroanatomic observations of the brain in autism. In M. L. Bauman, \& T. L. Kemper (Eds.), The neurobiology of autism. (pp. 119-145). Baltimore: John Hopkins University Press.

Berthier, M. L., Starkstein, S. E., \& Leiguarda, R. (1990). Developmental cortical anomalies in Asperger's syndrome: neuroradiological findings in two patients. Journal of Neuropsychiatry and Clinical Neurosciences, 2, 197-201.

Bertone, A., Mottron, L., Jelenic, P., \& Faubert, J. (2003). Motion perception in autism: A "Complex" Issue. Journal of Cognitive Neuroscience, 15, 1-8.

Blake, R., Turner, L. M., Smoski, M. J., Pozdol, S. L., \& Stone, W. L. (2003). Visual recognition of biological motion is impaired in children with autism. Psychological Science, 14(2), 151-157.

Britten, K. H., Shalden, M. N., Newsome, W. T., \& Movshon, J. A. (1992). The analysis of visual motion: a comparison of neuronal and psychophysical performance. Journal of Neuroscience, 12, 4745-4765.

Brown, W. M., Hines, M., Fane, B. A., \& Breedloves, S. M. (2002). Masculinized finger length patterns in human males and 
females with congenital adrenal hyperplasia. Hormones and Behaviour, 42, 380-386.

Chaudhuri, A. (1990). Modulation of the motion aftereffect by selective attention. Nature, 344, 60-62.

Cody, H., Pelphrey, K., \& Piven, J. (2002). Structural and functional magnetic resonance imaging of autism. International Journal of Developmental Neuroscience, 20, 421-438.

Courchesne, E. (1997). Brainstem, cerebellar and limbic neuroanatomical abnormalities in autism. Current Opinion in Neurobiology, 7, 269-278.

Courchesne, E., Townsend, J., Akshoomoff, N. A., Saitoh, O., Yeung-Courchesne, R., Lincoln, A. J., James, H. E., Haas, R. H., Schreibman, L., \& Lau, L. (1994). Impairment in shifting attention in autistic and cerebellar patients. Behavioural Neuroscience, 108, 848-865.

Dow, R., \& Moruzzi, G. (1958). The physiology and pathology of the cerebellum. Minneapolis: University of Minnesota Press.

Eden, G., Vanmeter, J., Rumsey, J., Maisog, J., Woods, R., \& Zeffiro, T. (1996). Abnormal processing of visual motion in dyslexia revealed by functional brain imaging. Nature, 382, 222-233.

Fawcett, A. J., Nicolson, R. I., \& Dean, P. (1996). Impaired performance of children with dyslexia on a range of cerebellar tasks. Annals of Dyslexia, 46, 259-283.

Galaburda, A. M., Menard, M. T., \& Rosen, G. D. (1994). Evidence for aberrant auditory anatomy in developmental dyslexia. Proceedings of the National Academy of Science, 91, 8010-8013.

Gepner, B., \& Mestre, D. (2002a). Brief Report: Postural reactivity to fast visual motion differentiates autistic children from children with Asperger syndrome. Journal of Autism and Developmental Disorders, 32, 231-238.

Gepner, B., \& Mestre, D. (2002b). Rapid visual-motion integration deficit in autism. Trends in Cognitive Sciences, 6, 455.

Gepner, B., Mestre, D., Masson, G., \& De-Schonen, S. (1995). Postural effects of motion vision in young autistic children. Neuroreport, 6, 1211-1214.

Geschwind, N., \& Galaburda, A. M. (1985). Cerebral Lateralization: Biological mechanisms, associations, and pathology: I. A hypothesis and a program for research. Archives of Neurology, $42,428-459$.

Ghaziuddin, M., Butler, E., Tsai, L., \& Ghaziuddin, N. (1994). Is clumsiness a marker for Asperger syndrome? Journal of Intellectual Disability Research, 38, 519-527.

Hansen, P. C., Stein, J. F., Orde, S. R., Winter, J. L., \& Talcott, J. B. (2001). Are dyslexics' visual deficits limited to measures of dorsal stream function? Neuroreport, 12, 1527-1530.

Henderson, S., \& Sugden, D. (1992). The movement assessment battery for children. Sidcup: The Psychology Corporation.

Herman, A. E., Galaburda, A. M., Fitch, R. H., Carter, A. R., \& Rosen, G. D. (1997). Cerebral Microgyria, thalamic cell size and auditory temporal processing in male and female rats. Cerebral Cortex, 7, 453-464.

Ivry, R. B., \& Diener, H. C. (1991). Impaired velocitiy perception in patients with lesions of the cerebellum. Journal of Cognitive Neuroscience, 3, 355-366.

Kondo, T., Zakany, J., Innis, J. W., \& Duboule, D. (1997). Of fingers, toes and penises. Nature, 390, 185-198.

Livingstone, M. S., Rosen, G. D., Drislane, F. W., \& Galaburda, A. M. (1991). Physiological and anatomical evidence for a magnocellular deficit in developmental dyslexia. Proceedings of the National Academy of Science, 88, 7943-7949.

Lovegrove, W., Bowling, A., Badcock, B., \& Blackwood, M. (1980). Specific reading disability: differences in contrast sensitivity as a function of spatial frequency. Science, 210, 439-440.

Lyman, H. B. (1978). Test scores and what they mean. Englewood Cliffs, NJ: Prentice Hall, Inc.

Manjiviona, J., \& Prior, M. (1995). Comparison of Asperger syndrome and high-functioning autistic children on a test of motor impairment. Journal of Autism and Developmental Disorders, $25,23-39$.

Manning, J. T. (2002). Digit ratio: A pointer to fertility, behaviour and health. New Brunswich NJ: Rutgers University Press.

Manning, J. T., Baron-Cohen, S., Wheelwright, S., \& Sanders, G. (2001). The 2nd to 4th digit ratio and autism. Developmental Medicine \& Child Neurology, 43, 160-164.

Manning, J. T., Scutt, D., Wilson, J., \& Lewis-Jones, D. I. (1998). The ratio of 2 nd to 4 th digit length: a predictor of sperm numbers and concentrations of testosterone, lutenizing hormone and oestrogen. Human Reproduction, 13, 3000-3004.

McFadden, D., \& Bracht, M. S. (2003). The relative lengths and weights of metacarpals and metatarsals in baboons (Papio hamadryas). Hormones and Behaviour, 43, 347-355.

Milne, E., Swettenham, J., Hansen, P., Campbell, R., Jeffries, H., \& Plaisted, K. (2002). High motion coherence thresholds in children with autism. Journal of Child Psychology and Psychiatry, 43, 255-263.

Nawrot, M., \& Rizzo, M. (1995). Motion perception deficits from midline cerebellar lesions in human. Vision Research, 35, 723-731.

Newsome, W. T., \& Pare, E. B. (1988). A selective impairment of motion perception following lesions of the middle temporal area (MT). Journal of Neuroscience, 8, 2201-2211.

O'Brien, J., Spencer, J., Atkinson, J., Braddick, O., \& Wattam-Bell, J. (2002). Form and motion coherence processing in dyspraxia: evidence of a global spatial processing deficit. Neuroreport, 113, 1399-1402.

Ökten, A., Kalyoncu, M., \& Yaris, N. (2002). The ratio of secondand fourth- digit lengths and congenital adrenal hyperplasia due to 21-hyroxylas deficiency. Early Human Development, 70, $47-54$.

Otsuka, H., Harada, M., Mori, K., Hisaoka, S., \& Nishitani, H. (1999). Brain metabolites in the hippocampus-amygdala region and cerebellum in autism: an $1 \mathrm{H}-\mathrm{MR}$ spectroscopy study. Neuroradiology, 41, 517-519.

Phelps, V. R. (1952). Relative index finger length as a sex-influenced trait in man. American Journal of Human Genetics, 4, 72-89.

Piven, J., Berthier, M. L., Starkstein, S. E., Nehme, E., Pearlson, G., \& Folstein, S. (1990). Magnetic resonance imaging evidence for a defect of cerebral cortical development in autism. American Journal of Psychiatry, 147, 734-739.

Ramus, F. (2002). Evidence for a domain-specific deficit in developmental dyslexia. Behavioral and Brain Sciences, 25, 767-768.

Ramus, F. (2003). Developmental dyslexia: specific phonological deficit or general sensorimotor dysfunction? Current Opinion in Neurobiology, 13, 212-218.

Ramus, F., Rosen, S., Dakin, S. C., Day, B. L., Castellote, J. M., White, S., \& Frith, U. (2003). Theories of developmental dyslexia: insights from a multiple case study of dyslexic adults. Brain, 126, 841-865.

Raven, J., Court, J., \& Raven, J. (1988). Raven's standard progressive matrices. London: H. K. Lewis \& Co.Ltd.

Rose, D., Bradshwaw, M. F., \& Hibbard, P. B. (2003). Attention affects the stereoscopic depth aftereffect. Perception, 32, 635-640.

Rosen, G. D., Herman, A. E., \& Galaburda, A. M. (1999). Sex differences in the effects of early neocortical injury on neuronal size distribution of the medial geniculate nucleus in the rat are mediated by perinatal gonadal steroids. Cerebral Cortex, 9 , 27-34.

Skottun, B. C. (2000). The magnocellular deficit theory of dyslexia: the evidence from contrast sensitivity. Vision Research, 40, $111-127$.

Spencer, J., O’Brien, J., Riggs, K., Braddick, O., Atkinson, J., \& Wattam-Bell, J. (2000). Motion processing in autism: Evidence for a dorsal stream deficiency. Neuroreport, 11, 2765-2767.

Stein, J., \& Glickstein, M. (1992). Role of the cerebellum in visual guidance of movement. Physiology Review, 72, 972-1017. 
Stein, J., Talcott, J., \& Walsh, V. (2000). Controversy about the visual magnocellular deficit in developmental dyslexia. Trends in Cognitive Sciences, 4, 209-211.

Stein, J., \& Walsh, V. (1997). To see but not to read; the magnocellular theory of dyslexia. Trends in Neuroscience, 20, $147-152$.

Talcott, J., Hansen, P., Willis-Owen, C., McKinnel, I. W., Richardson, A. J., \& Stein, J. (1998). Visual temporal processing in adult dyslexics: evidence for M-pathway dysfunction. NeuroOpthalmology, 20(4), 187-201.

Tallal, P. (1980). Auditory temporal perception, phonics, and reading disabilities in children. Brain and Language, 9, 182-198.
Thier, P., Haarmeier, T., Treue, S., \& Barash, S. (1999). Absence of a common functional denominator of visual disturbances in cerebellar disease. Brain, 122, 2133-2146.

Tordjman, S., Anderson, G. M., McBride, P. A., Hertzig, M. E., Snow, M. E., Hall, L. M., Ferrari, P., \& Cohen, D. J. (1995). Plasma androgens in autism. Journal of Autism and Developmental Disorders, 25, 295-304.

Townsend, J., Courchesne, E., \& Egaas, B. (1996). Slowed orienting of covert visual-spatial attention in autism: specific deficits associated with cerebellar and parietal abnormality. Development and Psychopathology, 8, 563-584.

Wing, L. (1981). Asperger's syndrome: a clinical account. Psychological Medicine, 11, 115-129. 